\title{
Avaliação ECOTOXICOLÓgICA Do IMPACTO Do LIXIVIADO DE COCO SOBRE ARTEMIA SALINA
}

\author{
Sérgio Thode Filho ${ }^{1 *}$, Heider Alves Franco ${ }^{2}$
}

${ }^{1}$ Graduado em Administração de Empresas, Especialista em Engenharia da Qualidade, Mestre em Sistemas de Gestão, D. Sc. em Ciências. Professor no IFRJ, Campus Duque de Caxias. Av. República do Paraguai, 120 - Vila Sarapuí, Duque de Caxias - RJ.

${ }^{2}$ Engenheiro Agrônomo, M.Sc. em Agricultura Orgânica, Doutorando PPGMA-UERJ, Professor no IFRJ, Campus Pinheiral. Rua: José Breves, 550, Centro. Pinheiral/RJ.

*Autorpara correspondência: heider.franco@ifrj.edu.br

Recebido em 23 de fevereiro de 2018. Aceito em 15 de agosto de 2018. Publicado em 26 de dezembro de 2018.

Resumo - O Brasil é o quarto maior produtor mundial de coco Cocos nucifera, e o primeiro da América do Sul com 80\% da produção total. Tanto no comércio do coco in natura, como na industrialização (sucos, doces etc), resultam resíduos como a casca do coco. A falta de conhecimento sobre as propriedades físico-químicas desses é um dos fatores que contribuem para o descarte inadequado. Objetivou-se avaliar o impacto do lixiviado de coco sobre organismos aquáticos, utilizando a A. salina como organismo teste. Após a eclosão dos cistos, foram transferidos cerca de 10 nauplios de A. salina para tubos de ensaio de $15 \times 150 \mathrm{~mm}$ contendo $10 \mathrm{~mL}$ (água salgada e extrato lixiviado de coco). Foram utilizados sete tratamentos com quatro repetições. A CL50 foi obtida matematicamente na dose com 37,5\% de lixiviado. O extrato lixiviado do resíduo da casca de coco verde é classificado como um resíduo não perigoso e não inerte devido à sua capacidade de solubilização. Portanto, seu destino final deve empregar algumas medidas especiais de coleta e proteção do ambiente, sobretudo de corpos hídricos.

Palavras-chave: Impacto Ambiental, Resíduo sólido, Extrato Lixiviado, Concentração Letal.

\section{Ecotoxicological evaluation of the impact of Coconut LEACHing on ARTEMIA SALINA}

Abstract - Brazil is the fourth largest producer of coconuts Cocos nucifera in the world, and the first in South America, with $80 \%$ of total production. Both in the coconut trade in natura, as in the industrialization (juices, sweets, etc), there are residues as the coconut shell. The lack of knowledge about the physicochemical properties of these is one of the factors that contribute to the inappropriate disposal. The objective of this study was to evaluate the impact of coconut leachate on aquatic organisms, using $A$. salina as a test organism. After the hatching of the cysts, about 10 nauplii of $A$. salina were transferred to $15 \times 150 \mathrm{~mm}$ test tubes containing $10 \mathrm{~mL}$ of salt water and coconut leach extract. Seven treatments with four replicates were used. The LC50 was obtained mathematically in the dose with $37.5 \%$ of leachate. The leachate extract from the residue of the green coconut shell is classified as a non-hazardous and non-inert residue because of its solubility. Therefore, its final destination must employ some special measures of collection and protection of the environment, especially of water bodies.

Keywords: EnVtronmental Impact, Solid Waste, Leachate Extract, Lethal Concentration.

\section{Evaluación ecotoxicológica del impacto del lixiviado de Coco sobre ARTEMia $S_{\text {ALINA }}$}

Resumen - Brasil es el cuarto mayor productor mundial de coco Cocos nucifera, y el primero de Sudamérica con el 80\% de la producción total. Tanto en el comercio del coco in natura, como en la industrialización (jugos, dulces, etc.), resultan residuos como la cáscara del coco. La falta de conocimiento sobre las propiedades físico-químicas de estos es uno de los factores que contribuyen al desecho inadecuado. Se objetivó evaluar el impacto del lixiviado de coco sobre organismos acuáticos, utilizando la A. salina como organismo de prueba. Tras la eclosión de los quistes, se transfirieron cerca de 10 nauplios de 
A. salina a tubos de ensayo de 15 x $150 \mathrm{~mm}$ que contenían $10 \mathrm{Ml}$ (agua salada y extracto de lixiviado de coco). Se utilizaron siete tratamientos con cuatro repeticiones. La CL50 fue obtenida matemáticamente en la dosis con el 37,5\% de lixiviado. El extracto lixiviado del residuo de la cáscara de coco verde se clasifica como un residuo no peligroso y no inerte debido a su capacidad de solubilización. Por lo tanto, su destino final debe emplear algunas medidas especiales de recolección y protección del ambiente, sobre todo de cuerpos hídricos.

Palabras clave: Impacto Ambiental, Residuos sólidos, Extracto Lixiviado, Concentración Letal.

\section{INTRODUÇÃO}

No Brasil, projeta-se que foram produzidas 214.405 toneladas de lixo diariamente. Desse total, cerca de $91 \%$ é coletado, entretanto apenas 58,4\% tem destino adequado a aterros sanitários, ficando o restante encaminhado a lixões ou aterros controlados. Do total de municípios do país, aproximadamente $62 \%$ apresenta algum tipo de iniciativa de coleta seletiva. (ABRELPE 2017).

Um dos esforços empreendidos no país para dar conta desse e de outros problemas relativos aos Resíduos Sólidos Urbanos (RSU) foi com a aprovação da Lei no 12.305 que instituiu a Política Nacional de Resíduos Sólidos (PNRS) (Brasil 2010).

A produção mundial de coco (Cocos nucifera) foi de 60.713 .136 milhões de toneladas em 2011, sendo os principais produtores mundiais a Indonésia, Filipinas, Índia e Brasil, que continuaram como responsáveis pela produção 18, 15, 12 e 3 milhões de toneladas em 2013. O Brasil é o quarto maior produtor mundial, contudo na América do Sul, o país é responsável por 80\% da produção total do continente (Franco 2016; Martins et al. 2016). Em 2015 a produção nacional de coco, também chamado de coco-da-baía (Cocos nucifera), sofreu uma queda de aproximadamente 1 milhão de toneladas (IBGE 2016).

Tanto no comércio do coco verde (consumo in natura), como na industrialização da fruta (sucos, doces, etc), resultam subprodutos caracterizados como sendo a casca do coco e seus componentes. A falta de conhecimento sobre as propriedades físico-químicas dos subprodutos do coco pode ser um dos fatores que contribuem para o descarte inadequado desse material (Rosa et al. 2001).

A disposição e acúmulo desse material representa um problema para a gestão sanitária de diversas áreas rurais e urbanas além de ser impactante sob a ótica do gerenciamento quando disposto em aterros sanitários (Santos 2006), principalmente devido a decomposição completa ser lenta e podendo levar cerca de 8 anos (Carijó et al. 2002). Esse fato, associado à volumosa dimensão unitária, torna a casca do coco um material de difícil manutenção para gestão sanitária urbana.

Como toda matéria orgânica, as cascas de coco quando depositadas em aterros sanitários, em condições anaeróbicas, além de ocupar grandes espaços, sua decomposição natural é responsável pela produção e liberação de metano. Depois do dióxido de carbono, o metano é o segundo principal gás causador do efeito estufa, que contribui para o aquecimento global (Passos 2005).

Uma vez que os subprodutos do coco são dispostos a céu aberto, podem contaminar o solo, por em risco a saúde local, deteriorar a área e o ambiente (Silveira 2008). De acordo com a Lei 12.305, de 2 de agosto de 2010, que decreta Política Nacional de Resíduos Sólidos (PNRS) (BRASIL 2010), o subproduto do coco possui características para ter a destinação final ambientalmente adequada, entretanto, este acaba sendo erroneamente tratado como um rejeito.

Segundo Martins et al. (2016) após discutir vasta revisão sobre os problemas do pós-consumo do Coco no Brasil, poucos autores associam o desenvolvimento tecnológico para o aproveitamento dos subprodutos do coco a um problema de gestão ambiental rural e urbana (Silveira 2008; Rosa et al. 2011), o que remete a necessidade de ensaios para avaliar o impacto ambiental desse resíduo. 
Um poluente emergente é toda e qualquer substância química de origem sintética ou natural, ou algum microrganismo que não é, normalmente, identificado no ambiente, mas com potencial para entrar neste meio e provocar efeitos ecológicos adversos (Flaherty e Dodson 2005).

Para a análise dos efeitos tóxicos das substâncias ou misturas, descartadas/dispostas incorretamente e/ou que venham a ser utilizadas nas atividades humanas, devem ser realizados testes de toxicidade ou bioensaios que visam predizer o impacto potencial de um xenobiótico (agente tóxico) ao ambiente. Esses são ensaios laboratoriais realizados sob condições experimentais específicas e controladas, visando estimar a toxicidade de substâncias, efluentes agrícolas, industriais e domésticos, medicamentos, produtos químicos em geral e amostras ambientais (águas ou sedimentos), além de avaliar a resultante de seus efeitos sinérgicos e antagônicos (Flohr et al. 2005; Costa et al. 2008).

O teste de toxicidade com Artemia salina é um ensaio biológico considerado uma das ferramentas mais utilizadas para a avaliação preliminar de toxicidade (Alves et al. 2000).

A fim de estabelecer a toxicidade de novos produtos naturais, vários ensaios podem ser utilizados, como o ensaio de letalidade com o microcrustáceo Artemia salina, que foi desenvolvido para detectar compostos bioativos em extratos vegetais (Silva et al. 2005). O presente trabalho objetivou avaliar o impacto do lixiviado de coco sobre organismos aquáticos, utilizando a A.salina como organismo teste.

\section{Material e Métodos}

O extrato lixiviado foi preparado de acordo com a Associação Brasileira de Normas Técnicas 10.005 (ABNT 2004a). Para preparar o extrato lixiviado, foi transferido 50,0 g de resíduos da casca de coco verde para uma cuba de polietileno e tratadas com 1,0 L de solução de extração $(5,7 \mathrm{~mL}$ de solução glacial ácido acético, $64,3 \mathrm{~mL}$ de $\mathrm{NaOH}$ 1,0 mol / L $\mathrm{L}^{-1}$ e $930 \mathrm{~mL}$ de água Milli-Q). O material foi agitado por aproximadamente $18 \mathrm{~h}$ com um agitador rotativo a $30 \mathrm{rpm}$ (Nova Etica ${ }^{\circledR}$ modelo 430) à temperatura ambiente e depois filtrado através de uma membrana de $0,45 \mu \mathrm{m}$.

Para realizar os testes foram utilizados cistos de $A$. salina da marca $\mathrm{JBL}^{\circledR}$ com $95 \%$ de eclosão e validade até novembro/18.

Para eclosão dos cistos foram utilizados os procedimentos descritos na literatura (Meyer et al. 1982; Mclaughlin et al. 1995; Thode-Filho et al. 2017) com adaptações.

Após a eclosão, cerca de 10 náuplios de $A$. salina foram transferidos para tubos de ensaio de 15 x $150 \mathrm{~mm}$ contendo $10 \mathrm{~mL}$ (solução salina e amostras a serem testadas), nas seguintes doses do lixiviado: T1 $=3,125 \%$ lixiviado de coco $+96,875 \%$ água salina; $\mathrm{T} 2=6,25 \%$ lixiviado de coco $+93,75 \%$ água salina; $\mathrm{T} 3=12,5 \%$ lixiviado de coco $+87,5 \%$ água salina; $\mathrm{T} 4=25 \%$ lixiviado de coco $+75 \%$ água salina; $\mathrm{T} 5=50 \%$ lixiviado de coco $+50 \%$ água salina e T6 = 100\% lixiviado de coco. Para o grupo controle negativo (TC), utilizou-se 100\% de água salina. Todos os tratamentos foram realizados com quatro réplicas.

A morte do microcrustáceo é evidenciada pela sua sedimentação. Por se tratar de um crustáceo ativo em água salina, a falta de movimento e sedimentação são os indicadores de morte do mesmo. Após a exposição de 48 horas, calculou-se a porcentagem média de indivíduos mortos de acordo com cada dose testada. Adicionalmente, calculou-se a $\mathrm{CL}_{50-48 \text { horas }}$, determinando a função obtida a partir da correlação entre a porcentagem de indivíduos mortos/ sedimentados e os tratamentos com o lixiviado testado. Na sequência, substitui-se o valor de y por 0,5 e, através da curva de dose-resposta, calculou-se a $\mathrm{CL}_{50-48 \text { horas }}$. A função foi determinada com $95 \%$ de confiabilidade.

Após a avaliação obtiveram-se a média da variável e submeteu-se aos testes Lilliefors para determinação da Normalidade e Cochran-Bartlett para determinação da Homogeneidade. Os dados foram submetidos à análise de variância (ANOVA) e as médias foram comparadas pelo teste de Tukey a 5\% de probabilidade entre as mesmas (Costa Neto 1977; Miller e Miller 1993).

As análises foram realizadas com auxílio do programa Assistat 7.7 (Silva e Azevedo 2016) e os gráficos construídos utilizando o QtiPlot 2011. 


\section{Resultados e Discussão}

De acordo com a Norma Técnica NBR 10.004 Resíduo sólido - Classificação (ABNT, 2004b), o resíduo da casca do coco é classificado como: Classe II A, Não perigoso - Não inerte.

Os valores médios do percentual de indivíduos mortos em função dos diferentes tratamentos do lixiviado estão apresentados na figura 1. Verificou-se um aumento no percentual de mortalidade dos indivíduos conforme se aumentou a dose do lixiviado. Para os tratamentos T1 (3,125\%) e T2 (6,25\%) não foram observadas diferenças significativas. Os tratamentos T3 (12,5\%) e T4 (25\%) também não apresentaram diferença estatística significativa entre si, contudo diferem dos primeiros. Os tratamentos T5 (50\%) e T6 (100\% do lixiviado bruto) provocaram $60 \%$ e 100\% de mortalidade dos indivíduos, respectivamente. Foi observada uma relação quadrática entre os tratamentos com o lixiviado de resíduo da casca do coco e a morte da $A$. salina.

Figura 1: Percentual médio da mortalidade de $A$. salina em função as diferentes doses de lixiviado de coco. Médias seguidas pela mesma letra minúscula não diferem estatisticamente entre si pelo Teste de Tukey a $5 \%$ de probabilidade.

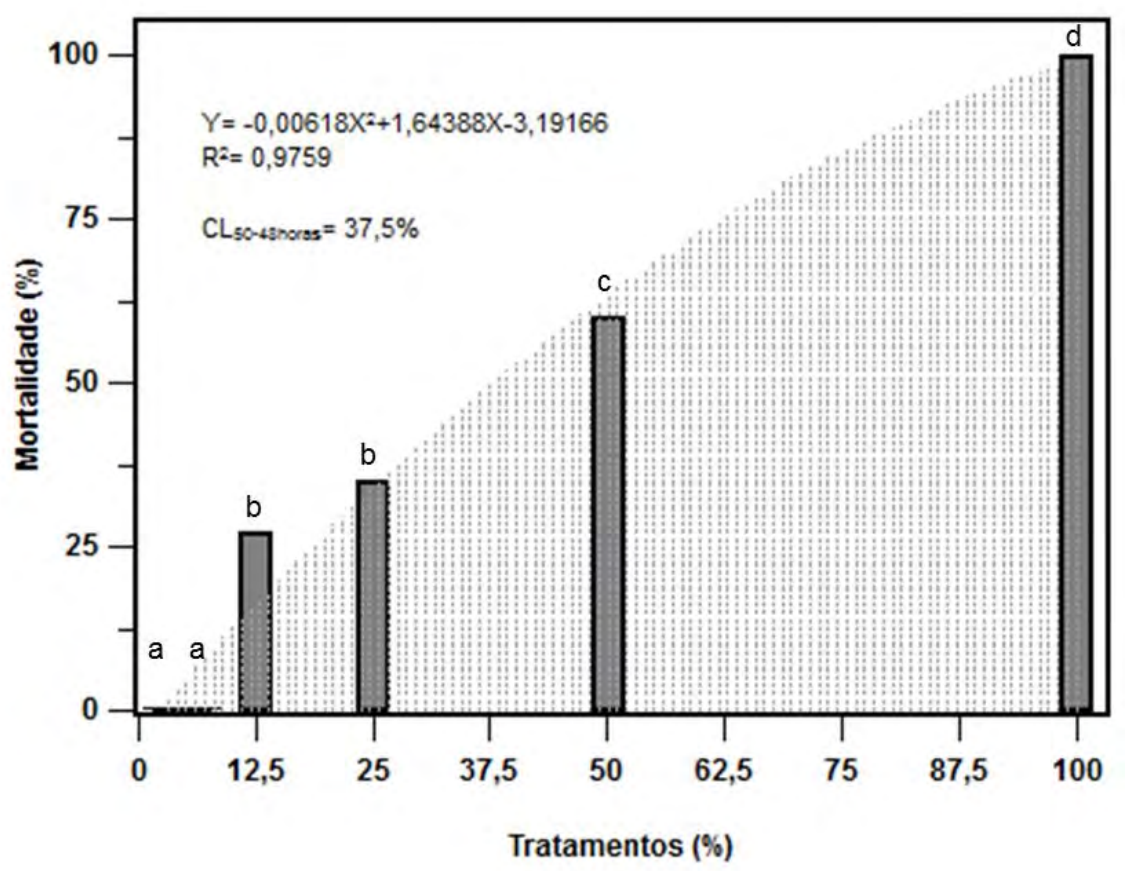

Para o cálculo da $\mathrm{CL}_{50-48 \text { horas }}$ do bioensaio, a função quadrática foi descrita por y $=-0,00618 \mathrm{x}^{2}+1,64388 \mathrm{x}$ 3,19166 com $\mathrm{R}^{2}=0,9759$ (Figura 1). Utilizando essa função e substituindo y por 50, encontrou-se o valor de $37,5 \%$ como sendo a dose de lixiviado $\left(\mathrm{CL}_{50-48 \text { horas }}\right.$ ) capaz de causar letalidade em 50\% dos organismos teste.

O lixiviado testado causou mortalidade no organismo teste, contudo não pode ser classificado como uma

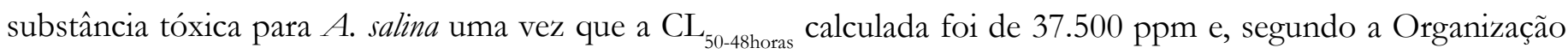
Mundial de Saúde (OMS), são consideradas tóxicas as substâncias que apresentam valores de $\mathrm{CL}_{50}$ abaixo de 1000 ppm em A. salina (Meyer et al. 1982).

Thode Filho et al (2017) verificou que a $\mathrm{CL}_{50-48 h o r a s}$ para $A$. salina com lixiviado de aterro sanitário (chorume) foi de 28,4\% (284.000 ppm). Segundo os autores a menor tolerância a este poluente pode estar relacionada aos parâmetros de salinidade, amônia e sulfatos encontrados. O resultado de 37,5\% de lixiviado de coco para a $\mathrm{CL}_{50}$ 48horas, apresenta uma maior tolerância do organismo teste, porém reforça-se a necessidade do tratamento e/ou reuso do material residual como forma de minimizar os possíveis impactos ao meio.

Adicionalmente, Lima et al. (2011) avaliaram a toxicidade de extratos do fruto de Euterpe edulis Martius frente à A.salina identificando que todos os extratos testados apresentaram $\mathrm{CL}_{50}$ superior a $1000 \mathrm{ppm}$ e $0 \%$ de mortalidade 
das artemias, indicando a não toxicidade dos extratos. Os dados obtidos pelos autores, corroboram com o presente estudo, indicando a não-toxicidade do lixiviado do casca de coco residual a partir das doses testadas.

Um poluente emergente é toda e qualquer substância química de origem sintética ou natural, ou algum microrganismo que não é, normalmente, identificado no ambiente, mas com potencial para entrar neste meio e provocar efeitos ecológicos adversos (Flaherty e Dodson 2005). De acordo com os autores, pode-se afirmar que o extrato lixiviado de Cocos nucifera não se apresentou como um potencial poluente de recursos hídricos a partir do contato $\operatorname{com}$ A. salina. Todavia cabe uma avaliação criteriosa, sobre a melhor forma de disposição do montante residual, pois o mesmo foi capaz de causar mortalidade no organismo teste.

Sendo assim, o extrato lixiviado do resíduo da casca do coco verde é classificado como um resíduo não perigoso e não inerte, devido à sua capacidade de solubilização. Principalmente devido ao tempo médio de 8 anos (Carijó et al. 2002), necessário para a completa decomposição da casca de coco, que além da solubilização esse processo é responsável pela produção e liberação de metano (Passos 2005). Portanto, seu destino final deve empregar medidas especiais de coleta e proteção do ambiente, sobretudo de corpos hídricos. Entende-se ainda, que a melhor destinação consiste no reaproveitamento do material residual em outras atividades, mitigando sempre que possível seu potencial poluidor.

\section{Conclusão}

Os tratamentos T1 e T2 não apresentaram mudança no comportamento fisiológico da $A$. salina a partir da introdução do poluente.

A CL $\mathrm{L}_{50-48 h o r a s}$ calculada para o poluente estudado foi encontrada no tratamento com $37,5 \%$ de lixiviado.

O extrato lixiviado do resíduo da casca de coco verde é classificado como um resíduo não perigoso e não inerte devido à sua capacidade de solubilização. Portanto, seu destino final deve empregar medidas especiais de coleta e proteção do ambiente, sobretudo de corpos hídricos.

\section{REFERÊNCIAS}

ABRELPE. ASSOCIAÇÃO BRASILEIRA DE EMPRESAS DE LIMPEZA PÚBLICA E RESÍDUOS ESPECIAIS. 2017. Panorama dos Resíduos Sólidos do Brasil. São Paulo: ABRELPE.

Alves TMA, Silva AF, Brandão M, Grandi TSM, Smânia EFA, Smânia Jr A, Zani CL. 2000. Biological screening of Brazilian medicinal plants. Memórias do Instituto Oswaldo Cruz, 95: 367-373.

ABNT. ASSOCIAÇÃO BRASILEIRA DE NORMAS TÉCNICAS. 2004a. NBR 10.005: Procedimento para obtenção de extrato lixiviado de Resíduos Sólidos. Rio de Janeiro.

ABNT. ASSOCIAÇÃO BRASILEIRA DE NORMAS TÉCNICAS. 2004b. NBR 10.004: Resíduos sólidos - Classificação. Rio de Janeiro.

BRASIL. 2010. Política Nacional de Resíduos Sólidos. Lei no 12.305, de 2 de agosto de 2010. Disponível em: http://www. planalto.gov.br/ccivil_03/_ato2007-2010/2010/lei/112305.htm. Acesso em: 13 fev. 2018.

Carijó OA, Liz RS, Makashima N. 2002. Fibra da casca do coco-verde como substrato agrícola. Horticultura Brasileira. v. 4, n. 20, p. 533-535. 
Costa CR, Olivi P, Botta CM, Espindola ELG. 2008. A toxicidade em ambientes aquáticos: discussão e métodos de avaliação. Quimica Nova. V. 31 n. 7, p. 1820-1830.

Costa Neto PLO. 1977. Estatística. São Paulo: Edgard Blücher, 264p.

Flohr L, Brentano DM, Carvalho-Pinto CRS, Machado VG, Matias WG. 2005. Classificação de resíduos sólidos industriais com base em testes ecotoxicológicos utilizando Daphnia magna: Uma alternativa. Biotemas, 18:7-18.

Flaherty CM, Dodson SI. Effects of pharmaceuticals on Daphnia survival, growth, and reproduction. Chemosphere, v. 61, n. 2, p. 200-207, 2005.

Franco HA. 2016. Introdução à Fruticultura. 1. ed. Curitiba: Livro Técnico, 180p. ISBN: 978-85-8409-071-6.

IBGE. 2016. Levantamento Sistemático da Produção Agrícola: pesquisa mensal de previsão e acompanhamento das safras agrícolas no ano civil. Rio de Janeiro: Instituto Brasileiro de Pesquisa Estatística, 78 p. v. 29, n. 01.

Lima CP de, Cunico MM, Trevisan RR, Philippsen AF, Miguel OG, Miguel MD. 2011. Efeito alelopático e toxicidade frente à Artemia salina Leach dos extatos do fruto de Euterpe edulis Martius. Acta Botanica Brasilica, 25(2), 331-336. https:// dx.doi.org/10.1590/S0102-33062011000200009

Martins AP, Silva PLR da, Watanabe T, Borelli C, Marcicano JPP, Sanches RA. 2016. O Problema do Pós-consumo do Coco no Brasil: Alternativas e Sustentabilidade. Sustentabilidade em Debate - Brasília, v. 7, n. 1, p. 44-57, jan/abr.

Mclaughlin JL, Saizarbitori T-C, Anderson JE. 1995. Tres bioensayos simples para quimicos de productos naturales. Rev Soc Venez Quim 18: 13-18.

Meyer BN, Ferrigni NR, Putnam JE, Jacobsen LB, Nichols DE, Mclaughlin JL. 1982. Brine shrimp, a convenient general bioassy for active-plant constituents. Planta Med 45: 31-34.

Miller JC, Miller JN. 1993. Statistics for analytical chemistry. 3. ed. Chichester: Ellis Horwood, 233p.

Passos PRA. 2005. Destinação Sustentável de Cascas de Coco (Cocos nucifera) Verde: Obtenção de Telhas e Chapas de Partículas. 2005. 186 f. Tese (Doutorado em Ciências e Planejamento Energético), Universidade Federal do Rio de Janeiro, Rio de Janeiro, 2005.

Rosa MF, Santos FJS, Montenegro AAT, Abreu FAP, Correia D, Araujo FBS, Noroes ERV. 2001. Caracterização do pó da casca de coco-verde usado como substrato agrícola. Embrapa Agroindustrial Tropical. Comunicado Técnico.

Rosa MF, Souza Filho MSM, Figueiredo MCB, Morais JPS, Santaella ST, Leitão RC. 2011. Valorização de resíduos da agroindústria. In: II Simpósio Internacional sobre Gerenciamento de Resíduos Agropecuários e Agroindústria - II SIGERA, 2011, Foz do Iguaçu. Anais do II Simpósio Internacional sobre Gerenciamento de Resíduos Agropecuários e Agroindústria - II SIGERA, p. 89-105.

Santos AM. 2006. Estudo de Compósitos Híbridos Polipropileno / Fibras de Vidro e Coco para Aplicações em Engenharia. 2005. 90 p. Dissertação (Mestrado em Engenharia). Universidade Federal do Paraná, Curitiba.

Silva F de AS, AZEVEDO, C. A. V. de. The Assistat Software Version 7.7 and its use in the analysis of experimental data. Afr. J. Agric. Res, v.11, n.39, p.3733-3740, 2016. DOI: 10.5897/AJAR2016.11522. 
Silva TMS, Batista MM, Camara CA, Agra MF. 2005. Molluscicidal activity of some Brazilian Solanum spp. (Solanaceae) against Biomphalaria glabrata. Ann Trop Med Parasit 4: 419-425.

Silveira MS. 2008. Aproveitamento das cascas de coco-verde para produção de briquetes em Salvador-BA. 167 p. Dissertação (Mestrado em Gerenciamento e Tecnologias Ambientais no Processo Produtivo - Ênfase em Produção Limpa). Escola Politécnica Federal da Bahia, Salvador.

Thode Filho S, Jorge ENDLF, Oliveira, AS de, Marques MRC, Franco HA. 2017. Study on the use of Artemia salina as bioindicator in the ecotoxicological evaluation of landfill leachate. Electronic Journal of Management, Education and Environmental Technology (REGET), 21, 24-31p. 